OPEN ACCESS

Edited by:

Mustafa Naziroglu,

Süleyman Demirel University, Turkey

Reviewed by:

Felipe Simon,

Universidad Andrés Bello, Chile

Ana Gomis,

Consejo Superior de Investigaciones

Cientificas (CSIC), Spain

*Correspondence:

Takayuki Nakagawa

tknakaga@kuhp.kyoto-u.ac.jp

Specialty section:

This article was submitted to Membrane Physiology and Membrane

Biophysics,

a section of the journal

Frontiers in Physiology

Received: 20 June 2017 Accepted: 18 October 2017 Published: 01 November 2017

Citation:

Miyake T, Nakamura S, Meng Z, Hamano S, Inoue $K$, Numata $T$,

Takahashi N, Nagayasu $K$

Shirakawa H, Mori Y, Nakagawa T and Kaneko S (2017) Distinct Mechanism

of Cysteine Oxidation-Dependent Activation and Cold Sensitization of Human Transient Receptor Potential

Ankyrin 1 Channel by High and Low Oxaliplatin. Front. Physiol. 8:878. doi: 10.3389/fphys.2017.00878

\section{Distinct Mechanism of Cysteine Oxidation-Dependent Activation and Cold Sensitization of Human Transient Receptor Potential Ankyrin 1 Channel by High and Low Oxaliplatin}

Takahito Miyake ${ }^{1}$, Saki Nakamura', Zhao Meng ${ }^{1}$, Satoshi Hamano ${ }^{2}$, Keisuke Inoue ${ }^{2}$, Tomohiro Numata ${ }^{3}$, Nobuaki Takahashi' ${ }^{2}$ Kazuki Nagayasu ${ }^{1}$, Hisashi Shirakawa', Yasuo Mori ${ }^{2}$, Takayuki Nakagawa ${ }^{4 *}$ and Shuji Kaneko ${ }^{1}$

${ }^{1}$ Department of Molecular Pharmacology, Graduate School of Pharmaceutical Sciences, Kyoto University, Kyoto, Japan ${ }^{2}$ Department of Synthetic Chemistry and Biological Chemistry, Graduate School of Engineering, Kyoto University, Kyoto, Japan, ${ }^{3}$ Department of Physiology, Graduate School of Medical Sciences, Fukuoka University, Fukuoka, Japan, ${ }^{4}$ Department of Clinical Pharmacology and Therapeutics, Kyoto University Hospital, Kyoto, Japan

Oxaliplatin, a third-generation platinum-based chemotherapeutic agent, displays unique acute peripheral neuropathy triggered or enhanced by cold, and accumulating evidence suggests that transient receptor potential ankyrin 1 (TRPA1) is responsible. TRPA1 is activated by oxaliplatin via a glutathione-sensitive mechanism. However, oxaliplatin interrupts hydroxylation of a proline residue located in the N-terminal region of TRPA1 via inhibition of prolyl hydroxylase (PHD), which causes sensitization of TRPA1 to reactive oxygen species (ROS). Furthermore, PHD inhibition endows cold-insensitive human TRPA1 (hTRPA1) with ROS-dependent cold sensitivity. Since cysteine oxidation and proline hydroxylation regulate its activity, their association with oxaliplatin-induced TRPA1 activation and acquirement of cold sensitivity were investigated in the present study. A high concentration of oxaliplatin $(1 \mathrm{mM})$ induced outward-rectifier whole-cell currents and increased the intracellular $\mathrm{Ca}^{2+}$ concentration in hTRPA1-expressing HEK293 cells, but did not increase the probability of hTRPA1 channel opening in the inside-out configuration. Oxaliplatin also induced the rapid generation of hydrogen peroxide, and the resultant $\mathrm{Ca}^{2+}$ influx was prevented in the presence of glutathione and in cysteine-mutated hTRPA1 (Cys641Ser)-expressing cells, whereas proline-mutated hTRPA1 (Pro394Ala)-expressing cells showed similar whole-cell currents and $\mathrm{Ca}^{2+}$ influx. By contrast, a lower concentration of oxaliplatin $(100 \mu \mathrm{M})$ did not increase the intracellular $\mathrm{Ca}^{2+}$ concentration but did confer cold sensitivity on hTRPA1-expressing cells, and this was inhibited by PHD2 co-overexpression. Cold sensitivity was abolished by the mitochondria-targeting ROS scavenger mitoTEMPO and 
was minimal in cysteine-mutated hTRPA1 (Cys641Ser or Cys665Ser)-expressing cells. Thus, high oxaliplatin evokes ROS-mediated cysteine oxidation-dependent hTRPA1 activation independent of PHD activity, while a lower concentration induces cold-induced cysteine oxidation-dependent opening of hTRPA1 via PHD inhibition.

Keywords: TRPA1, oxaliplatin, reactive oxygen species, cold hypersensitivity, prolyl hydroxylase, cysteine oxidation, peripheral neuropathy

\section{INTRODUCTION}

Oxaliplatin (L-OHP), a third-generation platinum-based agent, is frequently used to treat locally advanced and metastatic cancers of the colon or rectum. However, it increases the incidence of chemotherapy-induced peripheral neuropathy $(\mathrm{CIPN})$, often resulting in chemotherapeutic dose delay or treatment discontinuation (Falcone et al., 2007; Miltenburg and Boogerd, 2014). In addition to cumulative and chronic CIPN after multiple chemotherapy cycles, oxaliplatin induces a peculiar acute CIPN, characterized by paresthesia, dysesthesia, or acral numbness, in $\sim 90 \%$ of patients during or within hours of infusion. Acute CIPN is specific to oxaliplatin and often triggered or exacerbated by cold exposure (Wilson et al., 2002; Miltenburg and Boogerd, 2014; Cavaletti and Marmiroli, 2015).

The mechanisms underlying L-OHP-induced chronic CIPN can be explained, at least in part, by neurotoxicity in peripheral sensory neurons due to mitochondrial dysfunction and generation of reactive oxygen species (ROS) (Joseph and Levine, 2009; Di Cesare Mannelli et al., 2012; Azevedo et al., 2013) following accumulation of platinum in the dorsal root ganglia (DRG) (Screnci et al., 2000; Cavaletti et al., 2001). By contrast, L-OHP-induced acute CIPN is recognized as a channelopathy. A body of evidence suggests that it is caused by alteration of the kinetics of the axonal voltage-gated $\mathrm{Na}^{+}$channel (Sittl et al., 2012; Deuis et al., 2013) and/or activation of transient receptor potential ankyrin 1 (TRPA1) (Nassini et al., 2011; Zhao et al., 2012).

TRPA1 is a polymodal cation channel that plays a pivotal role as a nociceptor (Wu et al., 2010; Viana, 2016). This channel is opened by a large number of irritant chemicals (Bandell et al., 2004; Jordt et al., 2004). TRPA1 is also activated by oxidative stimuli such as, ROS and hyperoxia (Takahashi et al., 2008, 2011). TRPA1 activation evoked by most irritant chemicals and oxidative stimuli is caused by covalent or oxidative modification of cysteine residues in the $\mathrm{N}$-terminal region (Hinman et al., 2006; Macpherson et al., 2007). On the other hand, we previously identified another mechanism for TRPA1 activation; a decrease in oxygen concentration diminishes the activity of prolyl hydroxylases (PHDs) and relieves TRPA1 from the PHD-dependent hydroxylation of a proline residue ( $\mathrm{Pro}^{394}$ ) located within the $\mathrm{N}$-terminal ankyrin repeat domain, leading to hypoxia-induced activation (Takahashi et al., 2011; So et al., 2016).

It is reported that both L-OHP and cisplatin activate TRPA1 via glutathione-sensitive mechanisms (Nassini et al., 2011). However, we previously demonstrated that L-OHP and its characteristic metabolite oxalate enhance the responsiveness of TRPA1, which may contribute to the cold hypersensitivity induced by L-OHP in mice, but this is not the case for cisplatin and paclitaxel (Zhao et al., 2012). We also demonstrated the molecular mechanism: L-OHP and oxalate inhibit PHD activity, which augments the sensitivity of human TRPA1 (hTRPA1) to ROS by inhibiting hydroxylation of Pro ${ }^{394}$. Furthermore, we found that use of a PHD inhibitor or a hTRPA1 mutant lacking the hydroxylation-susceptible Pro $^{394}$ residue induces hTRPA1 sensitization to ROS, which enables cold-insensitive hTRPA1 to sense cold by detecting cold-evoked ROS production (Miyake et al., 2016). Therefore, the cold-induced indirect activation of hTRPA 1 that is sensitized by PHD inhibition may be responsible for L-OHP-induced acute CIPN triggered by cold, although whether L-OHP actually endows cold sensitivity to hTRPA1expressing cells has not been clarified. Thus, L-OHP is likely to activate and sensitize TRPA1, but whether the L-OHP-induced TRPA1 activation and sensitization is due to the oxidation of cysteine residues and/or inhibition of proline hydroxylation remains unknown.

In this study, we investigated whether and how cysteine oxidation and/or inhibition of proline hydroxylation contribute to the L-OHP-induced hTRPA1 activation and sensitization in vitro. A high concentration of L-OHP evoked cysteine oxidationdependent hTRPA1 activation, independent of hydroxylation of the PHD-targeted proline residue, while a subthreshold concentration of L-OHP endowed hTRPA1 with cysteine oxidation-dependent cold sensitivity through PHD inhibition.

\section{MATERIALS AND METHODS}

\section{Reagents}

L-OHP and allyl isothiocyanate (AITC) were purchased from Wako Pure Chemical Industries (Osaka, Japan). N-tert-butyl- $\alpha$-phenylnitrone $(\mathrm{PBN})$, cremophore EL, 2aminoethoxydiphenyl borate (2-APB), poly-L-lysine, and D-mannitol were purchased from Sigma-Aldrich (St. Louis, MO). The 1,2-bis(2-aminophenoxy)ethane-N,N, $\mathrm{N}^{\prime}, \mathrm{N}^{\prime}$-tetraacetic acid (BAPTA) was acquired from Dojindo Laboratories (Kumamoto, Japan). The mitoTEMPO was obtained from Santa Cruz (Dallas, TX). Peroxy Green 1 (PG-1) was synthesized previously (Miyake et al., 2016) according to the literature (Miller et al., 2007). Other drugs and chemicals were obtained from Nacalai Tesuque (Kyoto, Japan).

\section{Plasmids}

Constructs consisting of recombinant hTRPA1, its cysteine mutants (C633S, C641S, C665S), its proline mutant (P394A), or the human PHD2 cDNA in the pCIneo expression vector were 
prepared previously (Takahashi et al., 2011). The pEGFP-C3 was purchased from Clontech Laboratories (Madison, WI).

\section{Cell Cultures and Transfection}

HEK293 cells were cultured in Dulbecco's modified Eagle's medium (DMEM) with GlutaMAX I (10566-016, Life Technologies) supplemented with $10 \%$ heat-inactivated fetal bovine serum (Sigma) and maintained at $37^{\circ} \mathrm{C}$ in a humidified incubator set at 5\% $\mathrm{CO}_{2}$. HEK293 cells were cotransfected with recombinant plasmids and pEGFP-C3 using SuperFect Transfection Reagent (Qiagen, Hilden, Germany) or Lipofectamine 2000 (Life Technologies). Two days after transfection, cells were placed onto coverslips coated with poly-L-lysine and used in electrophysiological recording or fluorometric imaging.

\section{Electrophysiology}

Electrophysiological recordings were performed with a pipette made from a glass capillary (outer diameter, $1.5 \mathrm{~mm}$ ) with an internal filament (Narishige, Tokyo, Japan) pulled using a P87 micropipette puller (Sutter, Novato, CA). Access resistance ranged from 2 to $5 \mathrm{M} \Omega$ when the pipette was filled with pipette solution described below. For whole-cell patch-clamp recordings, the bath solution contained $100 \mathrm{mM} \mathrm{NaCl}, 2 \mathrm{mM}$ $\mathrm{CaCl}_{2}$, and $10 \mathrm{mM}$ HEPES (adjusted to $\mathrm{pH} 7.4$ with $\mathrm{NaOH}$ and $300 \mathrm{mOsm}$ with $\mathrm{D}$-mannitol), and the pipette solution contained $100 \mathrm{mM}$ Cs-aspartate, $5 \mathrm{mM}$ BAPTA, $1.4 \mathrm{mM} \mathrm{Ca-}$ gluconate ( $30 \mathrm{nM}$ free $\mathrm{Ca}^{2+}$ ), $2 \mathrm{mM} \mathrm{MgSO}_{4}, 2 \mathrm{mM} \mathrm{MgCl}_{2}, 4 \mathrm{mM}$ $\mathrm{Na}_{2}$-ATP, $10 \mathrm{mM} \mathrm{Na} \mathrm{P}_{3} \mathrm{O}_{10}$, and $10 \mathrm{mM}$ HEPES (adjusted to $\mathrm{pH} 7.4$ with $\mathrm{CsOH}$ and 300 mOsm with D-mannitol). Currentvoltage relationships were measured using voltage ramps $(-100$ to $+100 \mathrm{mV}$ over $100 \mathrm{~ms}$ ) applied every $10 \mathrm{~s}$. The membrane potential was set at $0 \mathrm{mV}$. Access resistance values were compensated by $70 \%$. For inside-out patch-clamp recordings, the bath solution contained $50 \mathrm{mM}$ Cs-aspartate, $50 \mathrm{mM} \mathrm{CsCl}$, $10 \mathrm{mM}$ EGTA, $1 \mathrm{mM} \mathrm{CaCl} 2\left(10 \mathrm{nM}\right.$ free $\left.\mathrm{Ca}^{2+}\right), 1 \mathrm{mM} \mathrm{MgCl}_{2}$, $10 \mathrm{mM} \mathrm{Na}_{5} \mathrm{P}_{3} \mathrm{O}_{10}$, and $10 \mathrm{mM}$ HEPES (adjusted to $\mathrm{pH} 7.4$ with $\mathrm{CsOH}$ and $300 \mathrm{mOsm}$ with $\mathrm{D}$-mannitol), and the pipette solution contained $100 \mathrm{mM} \mathrm{CsCl}, 1 \mathrm{mM} \mathrm{MgCl} 2,1 \mathrm{mM}$ EGTA, and $10 \mathrm{mM}$ HEPES (adjusted to pH 7.4 with $\mathrm{CsOH}$ and 300 mOsm with D-mannitol). The membrane potential was set at $+80 \mathrm{mV}$. Data were filtered at $2.9 \mathrm{kHz}$. Experiments were conducted at room temperature. Patch-clamp recordings were performed using an EPC-10 patch-clamp amplifier (HEKA Instruments, Lambrecht, Germany) and PATCHMASTER software (HEKA). AITC $(100 \mu \mathrm{M})$ was used to validate the expression of hTRPA1. The representative trace was obtained at least three independent experiments.

\section{Measurement of Intracellular $\mathrm{Ca}^{2+}$ Concentration $\left(\left[\mathrm{Ca}^{2+}\right]_{\mathrm{i}}\right)$}

Cells on coverslips were loaded for 30-40 min with $5 \mu \mathrm{M}$ Fura2 acetoxymethyl ester (Fura-2 AM; Dojindo Laboratories) in Krebs-Ringer solution containing $140 \mathrm{mM} \mathrm{NaCl}, 5 \mathrm{mM} \mathrm{KCl}$, $1 \mathrm{mM} \mathrm{MgCl}_{2}, 2 \mathrm{mM} \mathrm{CaCl}_{2}, 10 \mathrm{mM}$ glucose, and $10 \mathrm{mM}$ HEPES ( $\mathrm{pH}$ adjusted to 7.4 with $\mathrm{NaOH}$ ) containing $0.005 \%$ cremophore EL. Fluorescence images were captured every $5 \mathrm{~s}$ using alternating excitation at 340 and $380 \mathrm{~nm}$ and emission at $510 \mathrm{~nm}$ with an AQUACOSMOS/ORCA-AG imaging system (Hamamatsu Photonics, Shizuoka, Japan). For the pretreatment, L-OHP $(100 \mu \mathrm{M})$ was added to the culture medium $2 \mathrm{~h}$ before loading. MitoTEMPO $(10 \mu \mathrm{M}, 10 \mathrm{mM}$ stock solution in DMSO was diluted with the Fura-2 contained Krebs-Ringer solution) was preloaded with Fura-2 loading. Note that all drugs used for pretreatments were removed by washing before $\mathrm{Ca}^{2+}$ imaging experiments. Experiments were conducted at room temperature unless otherwise stated. Cold stimulation was performed with an SC-20 dual in-line solution heater/cooler and a CL-100 temperature controller (Warner Instruments, Hamden, CT). The velocity of the cooling ramp is about $3.75^{\circ} \mathrm{C} / \mathrm{min}$. The ratio of the fluorescence intensity obtained by excitation/emission at $340 \mathrm{~nm} / 510 \mathrm{~nm}\left(\mathrm{~F}_{340}\right)$ to the fluorescence intensity obtained by excitation/emission at $380 \mathrm{~nm} / 510 \mathrm{~nm}\left(\mathrm{~F}_{380}\right)$, namely, $\mathrm{F}_{340} / \mathrm{F}_{380}$, was calculated to quantify the intracellular $\mathrm{Ca}^{2+}$ concentration $\left(\left[\mathrm{Ca}^{2+}\right]_{\mathrm{i}}\right)$. Cells with an $\mathrm{F}_{340} / \mathrm{F}_{380}$ ratio $>1.5$ at baseline were excluded. Statistical analysis of the change in the ratio, $\Delta$ Ratio $\left(\mathrm{F}_{340} / \mathrm{F}_{380}\right)$, was performed as follows; in Figure 3, the average $\Delta$ Ratio $\left(\mathrm{F}_{340} / \mathrm{F}_{380}\right)$ during $0-2 \mathrm{~min}$ after 2-APBapplication was used; in Figure 5, the $\Delta$ Ratio $\left(\mathrm{F}_{340} / \mathrm{F}_{380}\right)$ at 2 min after cold stimulation was used. AITC $(100 \mu \mathrm{M})$ or 2APB $(100 \mu \mathrm{M})$ was used to validate the expression of hTRPA1.

\section{Measurement of Intracellular $\mathrm{H}_{2} \mathrm{O}_{2}$ Level}

Intracellular $\mathrm{H}_{2} \mathrm{O}_{2}$ level was measured using PG-1, a fluorescent probe with high selectivity for $\mathrm{H}_{2} \mathrm{O}_{2}$ (Miller et al., 2007). Cells on coverslips were loaded for $30-40$ min with $5 \mu \mathrm{M}$ PG-1 in HEPES-buffered saline containing $107 \mathrm{mM} \mathrm{NaCl}, 6 \mathrm{mM} \mathrm{KCl}$, $1.2 \mathrm{mM} \mathrm{MgSO}_{4}, 2 \mathrm{mM} \mathrm{CaCl}_{2}, 11.5 \mathrm{mM} \mathrm{D}$-glucose, and $20 \mathrm{mM}$ HEPES (pH adjusted to 7.4 with $\mathrm{NaOH}$ ). Fluorescence images were captured every $20 \mathrm{~s}$ using alternating excitation at $488 \mathrm{~nm}$ and emission at $510 \mathrm{~nm}$ with the AQUACOSMOS/ORCA-AG imaging system (Hamamatsu Photonics). Experiments were conducted at room temperature. The fluorescence intensity obtained with excitation/emission of $488 \mathrm{~nm} / 510 \mathrm{~nm}$ relative to the values obtained at $0 \mathrm{~min}\left(\mathrm{~F} / \mathrm{F}_{0}\right)$ was calculated to validate the intracellular $\mathrm{H}_{2} \mathrm{O}_{2}$ concentration. The $\Delta \mathrm{F} / \mathrm{F}_{0}$ obtained from each cells at 15 min was used for statistical analysis.

\section{Statistical Analysis}

The data are presented as means \pm S.E.M. from $n$ independent experiments or cells. Statistical significances were calculated using GraphPad Prism 7 (GraphPad Software, La Jolla, CA). The data in Figures 2B,C, 3C,D, 5B,C were compared using unpaired Student's $t$-tests (vs. Ctrl in Figure 2C, vs. WT in Figures 3C,D, 5C). The data in Figures 1D, 5A were compared using one-way analyses of variance (ANOVA), followed by Tukey's multiple comparisons test. In all cases, $P<0.05$ were considered statistically significant.

\section{RESULTS}

\section{A High Concentration of L-OHP Induces hTRPA1 Activation}

To investigate whether L-OHP activates hTRPA1, we performed whole-cell patch-clamp recording and fura-2-based intracellular 
A

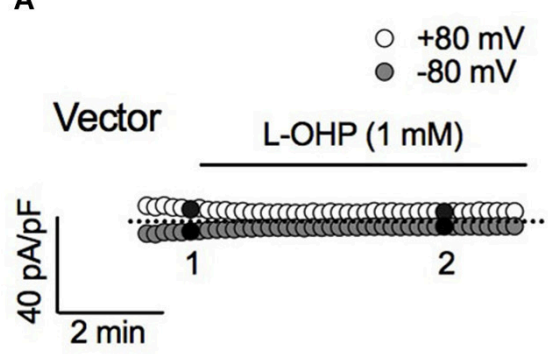

B

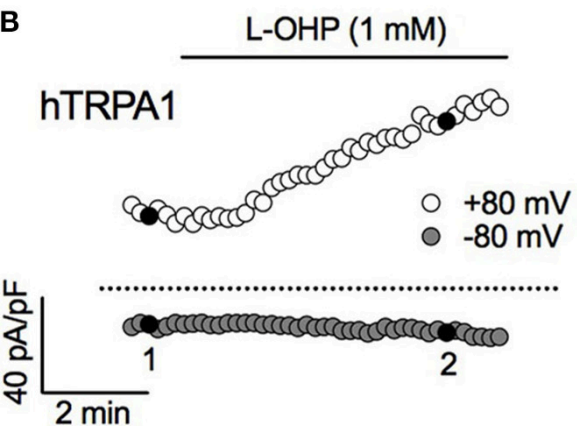

C

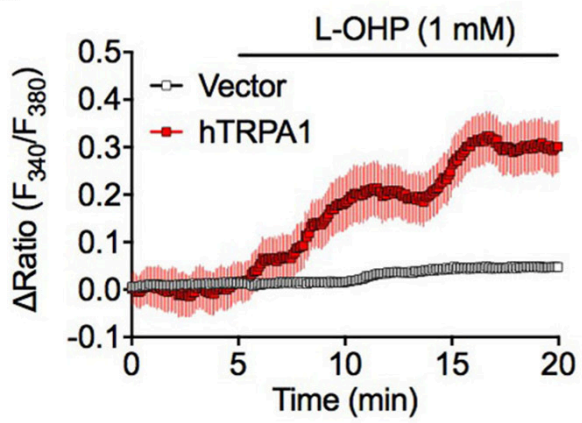

(pA/pF)
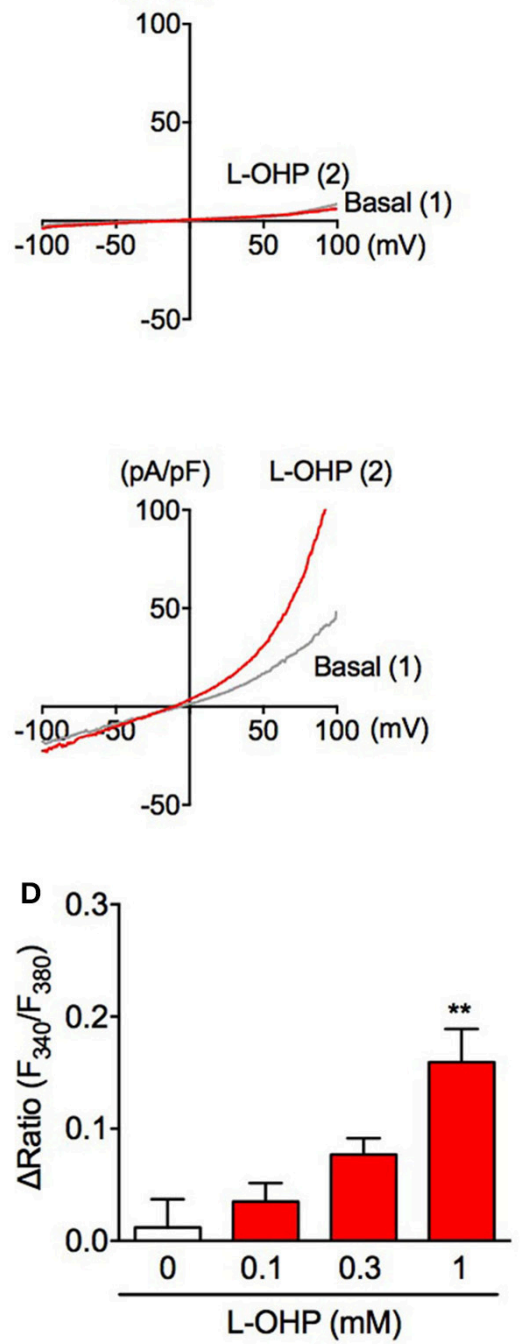

FIGURE 1 | High concentration of L-OHP induces hTRPA1 activation. (A,B) Effect of L-OHP (1 mM) on the whole-cell currents in HEK293 cells transfected with control (A) or hTRPA1 (B) vector. Left traces are representative whole-cell recordings. The current-voltage relationships were acquired at the time indicated by black-filled circle in (A,B), respectively. Membrane potential was set at $0 \mathrm{mV}$. (C) Effect of L-OHP on the intracellular Ca ${ }^{2+}$ concentration in HEK293 cells transfected with control or hTRPA1 vector ( $n=26-53$ cells). Left panel shows representative traces of intracellular $\mathrm{Ca}^{2+}$ imaging. Panel (D) shows the statistical analysis for concentration-dependent effect of L-OHP $(0.1,0.3$, and $1 \mathrm{mM}) . n=4-8$ independent experiments. ${ }^{* \star} P<0.01$ vs. the vehicle-treated hTRPA1-expressing cells (Veh). Panels (C,D) are expressed as mean \pm S.E.M. All of the experiments were performed at room temperature.

$\mathrm{Ca}^{2+}$ imaging experiments. In vector-transfected HEK293 cells, L-OHP (1 mM) did not induce a membrane current (Figure 1A). Meanwhile, in hTRPA1-expressing cells, L-OHP $(1 \mathrm{mM})$ induced gradually increasing TRPA1-like outwardrectifier currents (Figure 1B). Intracellular $\mathrm{Ca}^{2+}$ imaging revealed that L-OHP $(1 \mathrm{mM})$ also significantly increased $\left[\mathrm{Ca}^{2+}\right]_{\mathrm{i}}$ in hTRPA1-expressing cells in a concentrationdependent manner (Figure 1C). However, no statistically significant increase was observed at lower concentrations of L-OHP $(0.1$ or $0.3 \mathrm{mM})$. These results indicate that L-OHP activates hTRPA1 when present at a concentration of at least $1 \mathrm{mM}$.

\section{High L-OHP-Induced ROS Generation Is required for hTRPA1 Activation}

To clarify whether L-OHP activates hTRPA1 directly, we performed inside-out patch-clamp recording experiments for removing intracellular components. We held the membrane potential at $+80 \mathrm{mV}$, at which point weak voltage-gated hTRPA1 is easy to open. Although AITC $(100 \mu \mathrm{M})$ increased the probability of hTRPA1 opening, a high concentration of L-OHP (1 mM) had no effect (Figure 2A), suggesting that high L-OHP activates hTRPAl indirectly. Since L-OHP reportedly induces mitochondrial dysfunction and triggers ROS generation (Zheng et al., 2011), we examined the effect of high L-OHP (1 mM) on the 


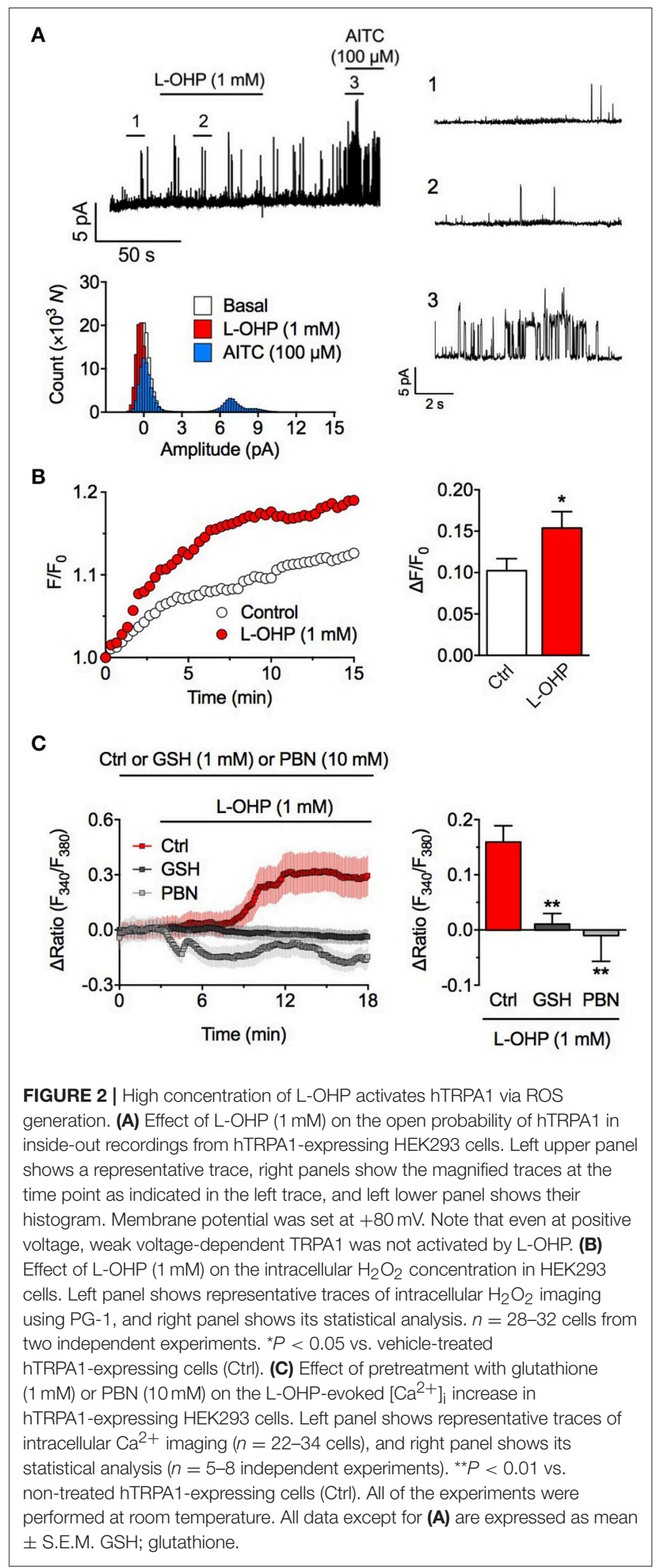

intracellular ROS level using the $\mathrm{H}_{2} \mathrm{O}_{2}$-specific indicator PG-1 (Miller et al., 2007). In HEK293 cells, high L-OHP induced $\mathrm{H}_{2} \mathrm{O}_{2}$ generation within $15 \mathrm{~min}$ (Figure 2B). Furthermore, the high
L-OHP-evoked $\left[\mathrm{Ca}^{2+}\right]_{i}$ increase was significantly suppressed in the presence of the antioxidants glutathione $(1 \mathrm{mM})$ or PBN $(10 \mathrm{mM})$ (Figure 2C). These results suggest that high LOHP does not directly activate hTRPA1 but rather triggers ROS generation, which causes glutathione-sensitive hTRPA1 activation.

\section{High L-OHP-Evoked hTRPA1 Activation Is Regulated by Cysteine Oxidation and Is Independent of PHDs}

Since ROS-induced TRPA1 activation is caused by oxidative modulation of the cysteine residues in the $\mathrm{N}$-terminal region of TRPA1 (Takahashi et al., 2008; Figure 3A), we compared the high L-OHP-evoked $\left[\mathrm{Ca}^{2+}\right]_{\mathrm{i}}$ increase in HEK293 cells expressing wild-type hTRPA1 (hTRPA1-WT) or hTRPA1 cysteine mutants (hTRPA1-C633S, C641S, C665S) in which each ROS or oxygensensitive cysteine residue was replaced with serine, which is a well-characterized strategy to investigate the redox sensitivity of cysteine residues in TRPA1 (Macpherson et al., 2007; Takahashi et al., 2008, 2011). Among the hTRPAl cysteine mutants, hTRPA1-C641S showed a significantly weaker response to high L-OHP (Figures 3B,C), while the responses of the other mutants (C633S and C665S) were comparable with hTRPA1-WT. By contrast, the response to 2-APB $(100 \mu \mathrm{M})$, a cysteine oxidationindependent TRPAl agonist (Hinman et al., 2006; Hu et al., 2009b), did not differ between hTRPA1-WT and its mutants (Figures 3B,D).

To investigate whether PHD inhibition is involved in high L-OHP-evoked hTRPA1 activation, we examined whether high L-OHP activates a PHD inhibition-insensitive hTRPA1 mutant hTRPA1-P394A. In whole-cell patch-clamp recordings, L-OHP $(1 \mathrm{mM})$ successfully induced TRPA1-like outwardrectifier currents in hTRPA1-P394A expressing cells (Figure 4A). In $\mathrm{Ca}^{2+}$ imaging experiments, both hTRPA1-WT and hTRPA1P394A showed a $\left[\mathrm{Ca}^{2+}\right]_{\mathrm{i}}$ increase induced by high L-OHP, and we did not observed any difference between them [Figure 4B, $n=6$ independent experiments, $P=0.233$ vs. hTRPA1-WT (Figure 1D, $1 \mathrm{mM}$ L-OHP treated group, $n=$ 8 independent experiments)]. These results suggest that high L-OHP activates hTRPA1 in a cysteine oxidation-dependent manner, while L-OHP-induced PHD inhibition is not involved in this phenomenon.

\section{A Low Concentration of L-OHP Endows Cold Sensitivity of hTRPA1 via Both PHD Inhibition and Cysteine Oxidation}

We recently reported that PHD inhibition causes hTRPA1 sensitization to ROS, which allows hTRPA1 to sense cold indirectly via cold-induced ROS generation (Miyake et al., 2016). Consistently, cold stimulation had minimal effect on $\left[\mathrm{Ca}^{2+}\right]_{\mathrm{i}}$ in non-treated hTRPA1-expressing cells in the present study, while pretreatment with a relatively low concentration of LOHP $(100 \mu \mathrm{M})$ for $2 \mathrm{~h}$ significantly increased $\left[\mathrm{Ca}^{2+}\right]_{\mathrm{i}}$ compared with that in non-treated hTRPA1-expressing cells. The coldevoked $\left[\mathrm{Ca}^{2+}\right]_{i}$ increase following low L-OHP pretreatment 

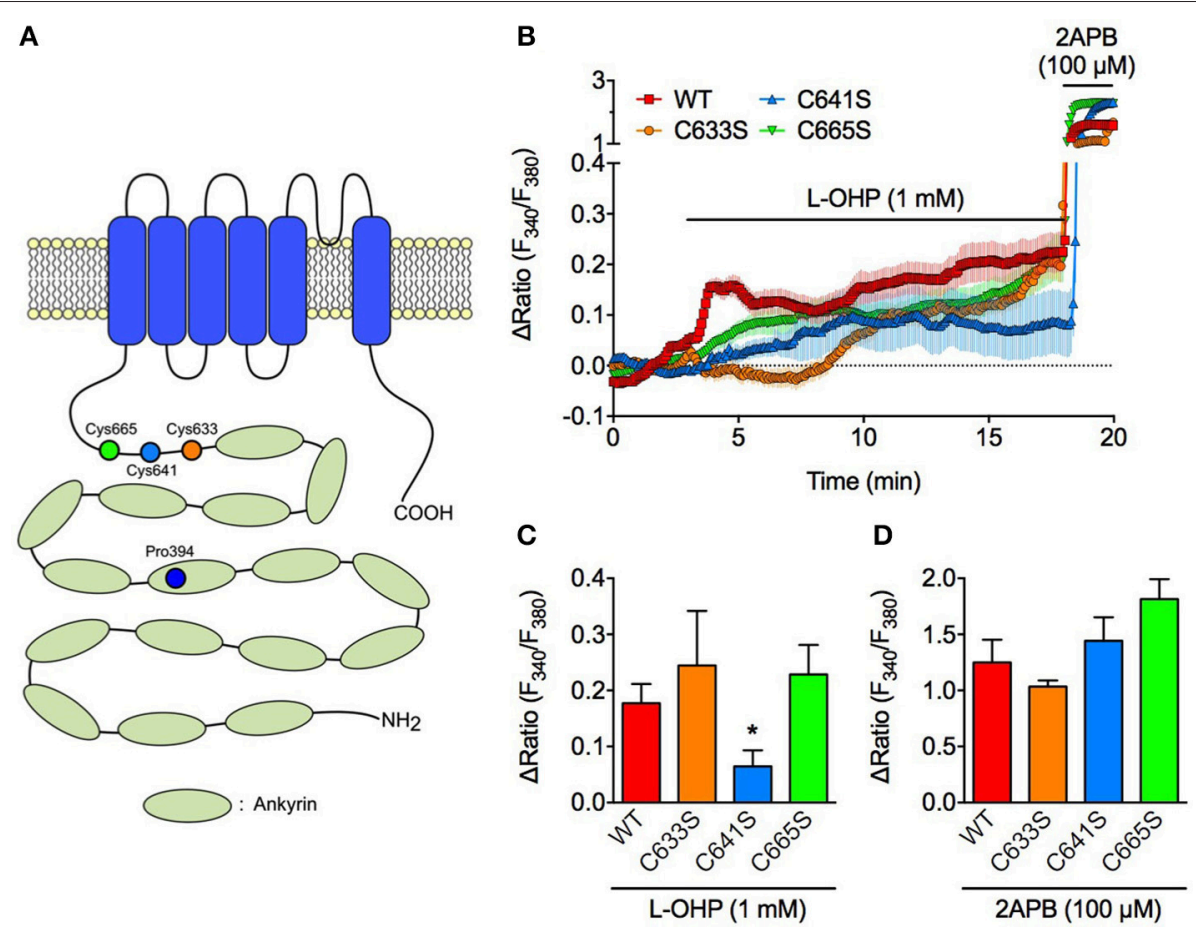

FIGURE 3 | High concentration of L-OHP activates hTRPA1 via Cys641 modification. Effect of L-OHP (1 mM) on the intracellular Ca ${ }^{2+}$ concentration in HEK293 cells expressing hTRPA1 cysteine mutants (hTRPA1-C633S, C641S, and C665S). 2-APB (100 $\mu \mathrm{M})$ was used to validate the expression of hTRPA1 cysteine mutants. (A) Schematic diagram illustrates the location of cysteine and proline residues of hTRPA1 that we focused in this study. (B-D) Representative traces of intracellular Ca ${ }^{2+}$ imaging ( $n=42-92$ cells) (B) and its statistical analysis for L-OHP- and 2-APB-evoked $\left[\mathrm{Ca}^{2+}\right]_{\mathrm{i}}$ increase (C,D) $(n=5-10$ independent experiments). Note that we used 2-APB instead of AITC, since AITC activates TRPA1 via Cys oxidation and not suitable for examining the full activation of TRPA1 in (D). ${ }^{*} P<0.05$ vs. wildtype hTRPA1-expressing cells (WT). All of the experiments were performed at room temperature. All data are expressed as mean \pm S.E.M.

was partially but significantly inhibited abolished in cells coexpressing hTRPA1 and PHD2 (Figure 5A) and in hTRPA1expressing cells pretreated with the mitochondria-targeting ROS scavenger mitoTEMPO $(10 \mu \mathrm{M}$; Figure 5B). To investigate whether hTRPA1 cysteine residues are involved in the low LOHP-endowed cold-evoked $\left[\mathrm{Ca}^{2+}\right]_{\mathrm{i}}$ increase, we performed the same experiments using hTRPA1 cysteine mutants. The coldevoked $\left[\mathrm{Ca}^{2+}\right]_{\mathrm{i}}$ increase following low L-OHP pretreatment was significantly smaller in hTRPA1-C641S and hTRPA1C665S mutants than that in hTRPA1-WT, while there was no difference between hTRPA1-WT and the hTRPA1-C633S mutant (Figure 5C). These results suggest that the low L-OHP-endowed cold sensitivity of hTRPA1 is dependent on both PHD inhibition and cysteine oxidation, and cold-induced mitochondrial ROS generation is important for the cold-evoked activation of hTRPA1 sensitized by low L-OHP.

\section{DISCUSSION}

Among others, TRPA1 is activated through dual mechanisms: covalent or oxidative modification of cysteine residues, and inhibition of hydroxylation of a proline residue in the $\mathrm{N}$ terminal region (Takahashi et al., 2011). The results of the present study showed that a high concentration of L-OHP $(\geq 1 \mathrm{mM})$ evoked hTRPA1 activation via ROS-mediated cysteine oxidation, independently of PHD inhibition, while both mechanisms are responsible for the cold-induced activation of hTRPA1 sensitized by the low concentration of L-OHP. The high concentration of L-OHP activated hTRPA1 in whole-cell patch clamp recordings and intracellular $\mathrm{Ca}^{2+}$ imaging experiments, but not membraneexcised inside-out patch clamp recordings, indicating that the high concentration of L-OHP affects cellular components other than hTRPA1 itself, and indirectly activates hTRPA1. Furthermore, glutathione and PBN suppressed the L-OHP ( $1 \mathrm{mM})$-induced activation of hTRPA1, suggesting that the high concentration of L-OHP induces ROS production that is followed by the activation of hTRPA1 (Figure 6). We previously reported that the low concentration of L-OHP increases the sensitivity of hTRPA1 to ROS via PHD inhibition (Miyake et al., 2016). In this study, we further found that the L-OHP $(100 \mu \mathrm{M})$ pretreated hTRPA1-expressing cells showed larger response to cold compared with the control hTRPA1-expressing cells, which was not observed when we used PHD2-overexpressing cells, and was partially inhibited by the pretreatment with mitoTEMPO. The L-OHP dependent cold response was suppressed in ROSsensitive cysteine-mutated hTRPA1-expressing cells, indicating that the pretreatment of L-OHP $(100 \mu \mathrm{M})$ allows hTRPA1 to sense cold in the same mechanisms we revealed before (Miyake et al., 2016) via modification to cysteine residues (Figure 6). 


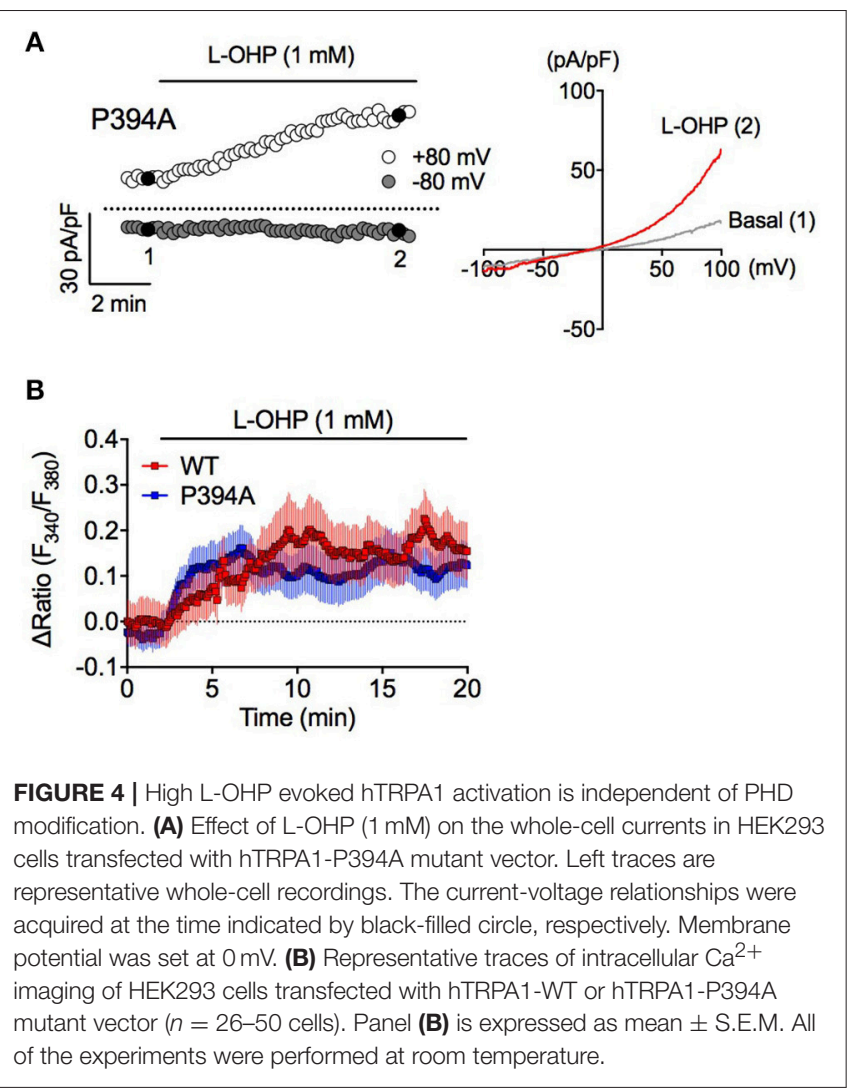

TRPA1 can be activated by heavy metals such as, gold, zinc, and cadmium via cysteine modification (Hu et al., 2009a; Gu and Lin, 2010; Hatano et al., 2013; Miura et al., 2013). However, the concentration of the platinum compound L-OHP required for hTRPAl activation $(1 \mathrm{mM})$ is much higher than that reported previously for other heavy metals $(\mu \mathrm{M})$ (Hu et al., 2009a; Hatano et al., 2013; Miura et al., 2013). The possibility that L-OHP directly activates hTRPA1 was ruled out since high L-OHP was unable to evoke TRPA1 activation in inside-out patch-clamp recording experiments. Rather, the results indicated indirect activation of hTRPA1 through L-OHP-induced ROS generation since (1) we detected an increase in intracellular $\mathrm{H}_{2} \mathrm{O}_{2}$ production following high L-OHP treatment, and (2) high LOHP-evoked hTRPAl activation was blocked by the membraneimpermeable antioxidant glutathione, consistent with a previous report (Nassini et al., 2011). It is reported that L-OHP induces apoptosis via mitochondrial damage (Gourdier et al., 2004), which triggers ROS generation (Bishop et al., 2010). Taken together, our findings suggest that high L-OHP triggers ROS generation, presumably by mitochondria, resulting in activation of TRPA1.

Cysteine oxidation is one of the most common mechanisms for TRPA1 activation by various agonists, and Cys633, Cys641, and Cys665 are crucial for activation by electrophiles (Hinman et al., 2006; Ibarra and Blair, 2013). Among these, Cys641 and Cys665 are important for activation by low concentrations of $\mathrm{H}_{2} \mathrm{O}_{2}$ (Takahashi et al., 2008) and nitric oxide (Kozai et al., 2014). Cys641 is also important for activation by zinc (Hu et al.,
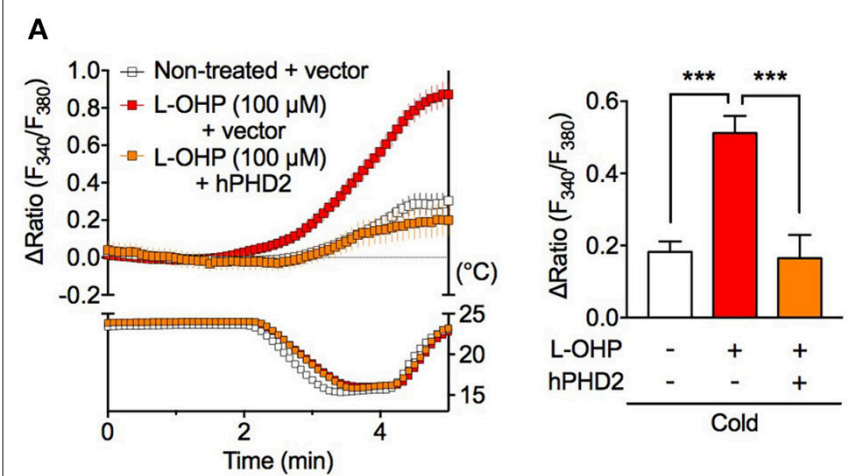

B
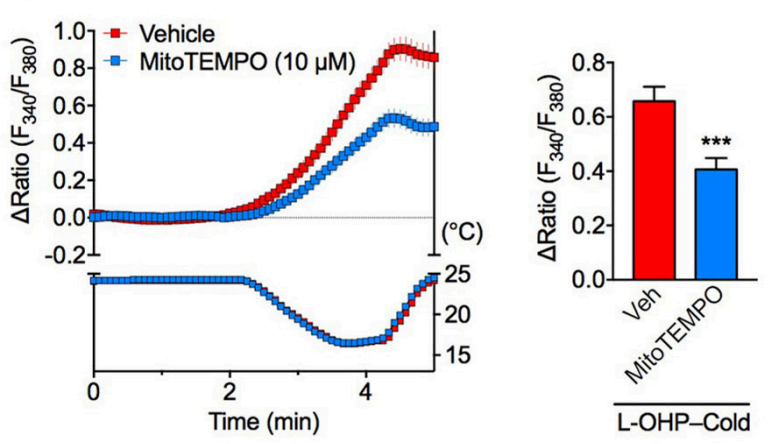

C
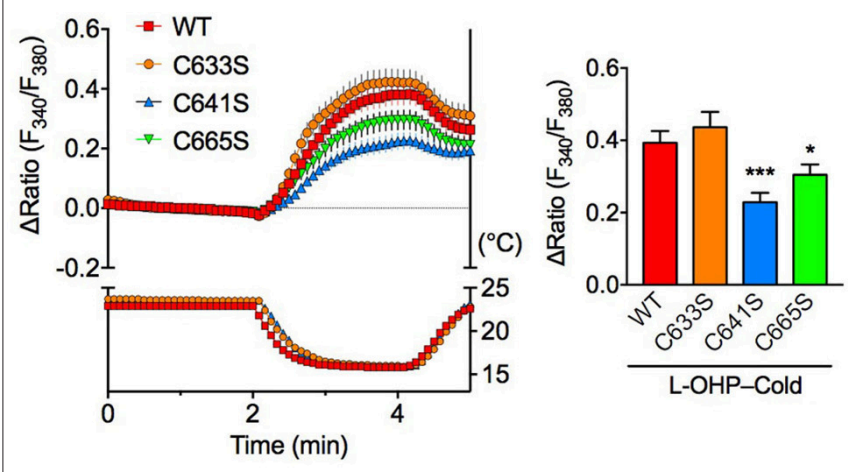

FIGURE 5 | Cold-induced activation of hTRPA1 sensitized by low L-OHP is dependent on PHD inhibition and cysteine oxidation. Effects of PHD2 overexpression (A), a mitochondria-targeted ROS scavenger (B) or hTRPA1 cysteine mutants $\mathbf{( C )}$ on the cold-evoked $\left[\mathrm{Ca}^{2+}\right]_{i}$ increase following pretreatment with L-OHP $(100 \mu \mathrm{M})$ for $2 \mathrm{~h}$ in hTRPA1-expressing HEK293 cells were investigated. (A) Left panel shows representative traces of intracellular $\mathrm{Ca}^{2+}$ imaging from hTRPA1-expressing HEK293 cells co-transfected with or without human PHD2 (top) and the temperature of the recording solution (bottom). Right panel shows its statistical analysis ( $n=47-83$ cells from two independent experiments). ${ }^{\star \star *} P<0.001$. (B) Left panel shows representative traces of intracellular $\mathrm{Ca}^{2+}$ imaging from L-OHP-treated hTRPA1-expressing HEK293 cells pretreated with vehicle or mitoTEMPO $(10 \mu \mathrm{M}$, loading with Fura-2 $\mathrm{Ca}^{2+}$ indicator; top) and the temperature of the recording solution (bottom). Right panel shows its statistical analysis ( $n=94-95$ cells from two independent experiments). ${ }^{\star * \star} P<0.001$ vs. vehicle $(0.1 \%$ DMSO)-treated cells (Veh). (C) Left panel shows representative traces of intracellular $\mathrm{Ca}^{2+}$ imaging from L-OHP-treated HEK293 cells expressing wildtype hTRPA1 (WT) or hTRPA1 cysteine mutants (hTRPA1-C633S, C641S, and C665S; top) and the temperature of the recording solution (bottom). Right panel shows its statistical analysis ( $n=202-266$ cells from three independent experiments). ${ }^{\star} P<0.05$, ${ }^{\star \star \star} P<0.001$ vs. WT. All data are expressed as mean \pm S.E.M. 


\section{A High L-OHP induced activation}

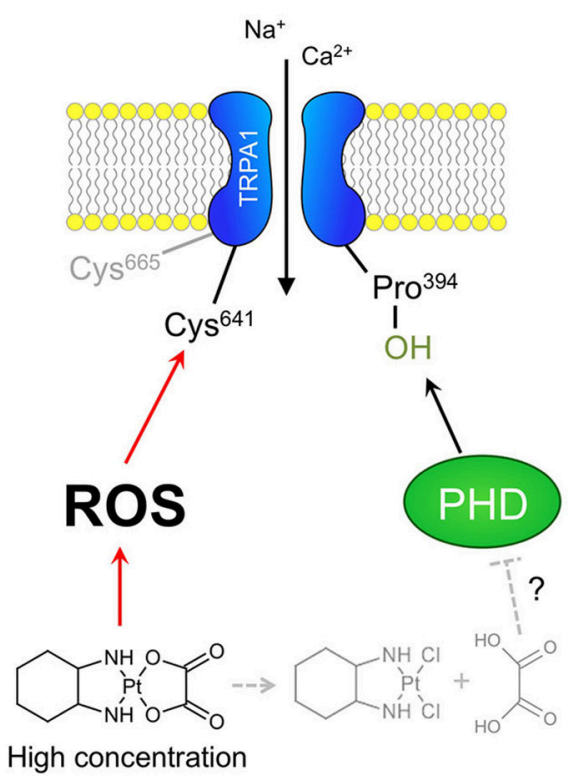

\section{B Low L-OHP mediated activation by cold}

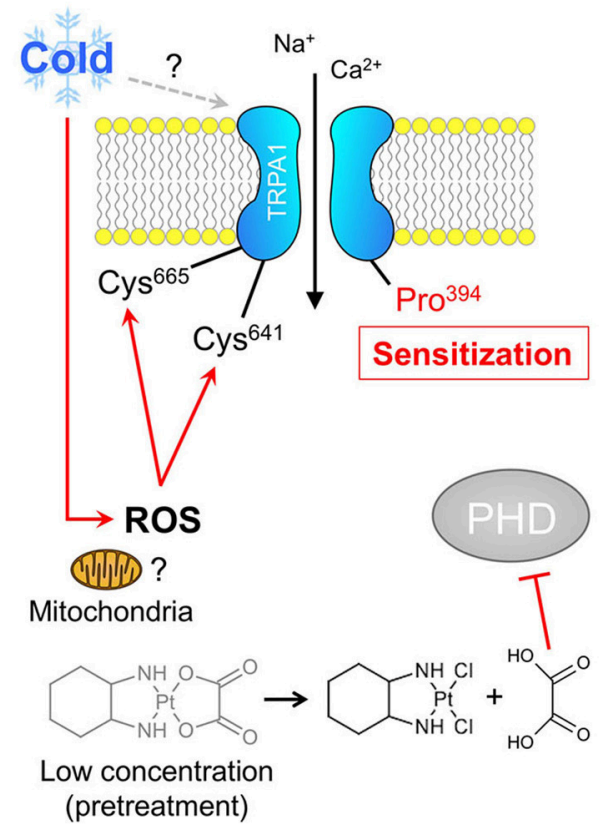

FIGURE 6 | Different concentration of L-OHP elicits cysteine oxidation-dependent activation or cold sensitization of hTRPA1. (A) The mechanism underlying the high L-OHP-induced hTRPA1 activation. L-OHP induces ROS generation (presumably via mitochondria), and the resultant ROS activate hTRPA1 via oxidation of Cys641. (B) The mechanism underlying the low L-OHP-mediated hTRPA1 activation by cold. The long exposure of L-OHP (more than $1 \mathrm{~h}$ ) causes the inhibition of PHDs by oxalate, a metabolite of L-OHP, which results in the sensitization of hTRPA1. The sensitized hTRPA1 is activated by ROS produced by mitochondria following cold exposure via oxidation of Cys 641 and Cys665.

2009a) and cadmium (Miura et al., 2013), whereas Cys633 is involved in activation by HNO (Eberhardt et al., 2014) and the gold compound auranofin (Hatano et al., 2013). In the present study, we showed that mutation of Cys641 inhibited high L-OHP-evoked hTRPA1 activation. This result further supports indirect hTRPAl activation by high L-OHP through ROS generation, although mutating Cys665 had no effect, which may partially contradict this finding. This paradox may indicate some additional roles of L-OHP in the high L-OHP-induced hTRPA1 activation, but further investigations are required. The fact that mutation of Cys633 did not affect high L-OHP-evoked hTRPA1 activation may suggest that platinum does not directly activate hTRPA1 in a cysteine-dependent manner, like gold.

Hydroxylation of the proline residue in the N-terminal region of TRPA1 by PHDs is critical for regulating TRPA1 activity (Miyake et al., 2016). Although L-OHP and its metabolite oxalate can inhibit PHDs (Miyake et al., 2016), the present results suggest that $\mathrm{PHDs}$ are not involved in the high LOHP-evoked hTRPA1 activation. This apparent discrepancy may be explained by the previous observation that induction of TRPA1 sensitization in mouse DRG neurons by low L-OHP pretreatment requires more than $1 \mathrm{~h}$ (Zhao et al., 2012). Thus, it is likely that inhibition of PHDs and induction of TRPA1 sensitization by L-OHP and/or oxalate may be slower than the high L-OHP-induced rapid ROS generation that activates TRPA1. It is probable that cysteine oxidation by ROS, rather than inhibition of proline hydroxylation by L-OHP and/or oxalate and subsequent delayed sensitization of hTRPA1, contributes to hTRPA1 activation evoked by high L-OHP.

We previously reported that PHD inhibition by a PHD inhibitor dimethyloxalylglycine (DMOG) sensitizes hTRPA1 to ROS and induces channel opening at cold temperatures. Furthermore, similar cold hypersensitivity is also observed in the mice treated with DMOG, which is also inhibited by a TRPA1 antagonist HC030031 (Miyake et al., 2016). Consistently, in the present study, pretreatment with a relatively low concentration of L-OHP potentiated the cold sensitivity of hTRPA1. Furthermore, this L-OHP-induced cold sensitivity was significantly reduced when treated with a mitochondriatargeting ROS scavenger, suggesting that ROS generated from mitochondria during cold exposure contributes to the L-OHPinduced cold sensitivity, similar to DMOG. Consistent with our results, previous in vivo experiments showed that L-OHPinduced cold hypersensitivity was attenuated by a single acute administration of a ROS scavenger (Miyake et al., 2016) or a mitochondria-targeting ROS scavenger (Toyama et al., 2014). Furthermore, the results obtained from Cys641 and Cys665 hTRPA1 mutants confirmed that these residues are responsible for activation by $\mathrm{H}_{2} \mathrm{O}_{2}$ (Takahashi et al., 2008) and contribute to the indirect cold sensitivity of hTRPA1 induced by low LOHP. These results suggest that ROS presumably generated from mitochondria during cold exposure oxidize cysteine residues in 
the N-terminal region of hTRPA1, thereby activating hTRPA1 following exposure to low L-OHP pretreatment. However, in this study, mitoTEMPO did not completely inhibit the coldinduced increase of $\left[\mathrm{Ca}^{2+}\right]_{\mathrm{i}}$ in the low L-OHP-treated hTRPA1 expressing cells. This mitoTEMPO-insensitive component may be ROS-independent but still PHD-dependent, since the overexpression of PHD2 completely inhibited the cold-induced hTRPA1 activation and hTRPA1-P394A mutant (that mimics a constitutively PHD-inhibited condition) shows a weak ROSindependent cold sensitivity (Miyake et al., 2016). By contrast, the mutation demonstrated that Cys633 had no effect on the indirect cold sensitivity of hTRPA1, which may indicate that platinum itself is not involved in this phenomenon. This hypothesis is consistent with our previous findings that other platinum-based chemotherapeutic agents such as, cisplatin and carboplatin do not induce acute cold hypersensitivity (Zhao et al., 2012).

The concentration of L-OHP in commercial infusions is about $1.25 \mathrm{mM}$, while the calculated blood concentration in patients is $<100 \mu \mathrm{M}$ (Chalret du Rieu et al., 2014). Although LOHP accumulates in the DRG and peripheral nerves (Screnci et al., 2000; Cavaletti et al., 2001), the concentration of L-OHP required to evoke TRPA1 activation $(1 \mathrm{mM})$ appears to be too high to explain L-OHP-induced acute CIPN. In addition, a high concentration of cisplatin, which does not induce acute CIPN, also activates TRPA1 via ROS generation (Nassini et al., 2011). Thus, high L-OHP-evoked TRPA1 activation via ROS generation is unlikely to be responsible for acute CIPN following L-OHP treatment. Interestingly, delayed mechanical, thermal, and cold hypersensitivity following repeated administration of L-OHP in rodents is prevented by some antioxidants (Joseph and Levine, 2009; Di Cesare Mannelli et al., 2012; Azevedo et al., 2013) and a TRPA1 blocker (Nassini et al., 2011). Furthermore, TRPA1 activation via ROS is associated with mechanical hypersensitivity induced by cisplatin (Nassini et al., 2011) and some other classes of chemotherapeutic agents such as, paclitacel (Materazzi et al., 2012), bortezomib (Trevisan et al., 2013), and vincristine (Old et al., 2014). Thus, ROS-mediated TRPA1 activation may be a common mechanism for cumulative and chronic CIPN.

\section{REFERENCES}

Azevedo, M. I., Pereira, A. F., Nogueira, R. B., Rolim, F. E., Brito, G. A., Wong, D. V., et al. (2013). The antioxidant effects of the flavonoids rutin and quercetin inhibit oxaliplatin-induced chronic painful peripheral neuropathy. Mol. Pain 9:53. doi: 10.1186/1744-8069-9-53

Bandell, M., Story, G. M., Hwang, S. W., Viswanath, V., Eid, S. R., Petrus, M. J., et al. (2004). Noxious cold ion channel TRPA1 is activated by pungent compounds and bradykinin. Neuron 41, 849-857. doi: 10.1016/S0896-6273(04)00 150-3

Bishop, N. A., Lu, T., and Yankner, B. A. (2010). Neural mechanisms of ageing and cognitive decline. Nature 464, 529-535. doi: 10.1038/nature08983

Cavaletti, G., Tredici, G., Petruccioli, M. G., Dondè, E., Tredici, P., Marmiroli, P. (2001). Effects of different schedules of oxaliplatin treatment on the peripheral nervous system of the rat. Eur. J. Cancer 37, 2457-2463. doi: 10.1016/S0959-8049(01)00300-8

Cavaletti, G., and Marmiroli, P. (2015). Chemotherapy-induced peripheral neurotoxicity. Curr. Opin. Neurol. 28, 500-507. doi: 10.1097/WCO. 0000000000000234
In conclusion, we further clarified the molecular details of how L-OHP activates or sensitizes hTRPA1. L-OHP exhibited complex concentration-dependent effects on hTRPA1; high L-OHP evoked hTRPA1 activation in a proline-independent manner, while low L-OHP sensitized hTRPA1 in a prolinedependent manner. This finding implies that the same chemical agent can function via different molecular mechanisms to regulate target proteins in a concentration-dependent manner. Nevertheless, the present results provide experimental evidence that TRPA1 blockage may be of clinical benefit for CIPN patients treated with L-OHP.

\section{AUTHOR CONTRIBUTIONS}

TM, TNa, and SK designed the project. TM, SN, ZM, SH, and KI performed the experiments. TM, SN, ZM, SH, HS, KN, and TNa analyzed the data; TNu, NT, and YM provided materials and technical advices. TM, TNa, and SK wrote the manuscript. SK supervised the experiments and finalized the manuscript.

\section{FUNDING}

This work was supported in part by Grants-in-Aid for Scientific Research (KAKENHI) from the Japanese Society for the Promotion of Science [Grants-in-Aid for Scientific Research (B) to $\mathrm{TNa}$ (26293019 and 17H04008) and SK (24390016), Challenging Exploratory Research to TNa (15K14961 and 17K19722), and Scientific Research on Innovative Area "Thermal Biology" to TNa (16H01386)] and by grants from the Salt Science Research Foundation (No. 14C4) and The Nakatomi Foundation. TM is a research fellow of Japan Society for the Promotion of Science.

\section{ACKNOWLEDGMENTS}

We thank M. Tominaga and K. Uchida for technical advices about how to manipulate the bath temperature in the $\mathrm{Ca}^{2+}$ imaging experiments.
Chalret du Rieu, Q., White-Koning, M., Picaud, L., Lochon, I., Marsili, S., Gladieff, L., et al. (2014). Population pharmacokinetics of peritoneal, plasma ultrafiltrated and protein-bound oxaliplatin concentrations in patients with disseminated peritoneal cancer after intraperitoneal hyperthermic chemoperfusion of oxaliplatin following cytoreductive surgery: correlation between oxaliplatin exposure and thrombocytopenia. Cancer Chemother. Pharmacol. 74, 571-582. doi: 10.1007/s00280-014-2525-6

Deuis, J. R., Zimmermann, K., Romanovsky, A. A., Possani, L. D., Cabot, P. J., Lewis, R. J., et al. (2013). An animal model of oxaliplatin-induced cold allodynia reveals a crucial role for Nav1.6 in peripheral pain pathways. Pain 154, 1749-1757. doi: 10.1016/j.pain.2013.05.032

Di Cesare Mannelli, L., Zanardelli, M., Failli, P., and Ghelardini, C. (2012). Oxaliplatin-induced neuropathy: oxidative stress as pathological mechanism. Protective effect of silibinin. J. Pain 13, 276-284. doi: 10.1016/j.jpain.2011.11.009

Eberhardt, M., Dux, M., Namer, B., Miljkovic, J., Cordasic, N., Will, C., et al. (2014). H2S and NO cooperatively regulate vascular tone by activating a neuroendocrine HNO-TRPA1-CGRP signalling pathway. Nat. Commun. 5, 4381. doi: $10.1038 /$ ncomms5381 
Falcone, A., Ricci, S., Brunetti, I., Pfanner, E., Allegrini, G., Barbara, C., et al. (2007). Phase III trial of infusional fluorouracil, leucovorin, oxaliplatin, and irinotecan (FOLFOXIRI) compared with infusional fluorouracil, leucovorin, and irinotecan (FOLFIRI) as first-line treatment for metastatic colorectal cancer: the Gruppo Oncologico Nor. J. Clin. Oncol. 25, 1670-1676. doi: 10.1200/JCO.2006.09.0928

Gourdier, I., Crabbe, L., Andreau, K., Pau, B., and Kroemer, G. (2004). Oxaliplatininduced mitochondrial apoptotic response of colon carcinoma cells does not require nuclear DNA. Oncogene 23, 7449-7457. doi: 10.1038/sj.onc.1208047

$\mathrm{Gu}$, Q., and Lin, R. L. (2010). Heavy metals zinc, cadmium, and copper stimulate pulmonary sensory neurons via direct activation of TRPA1. J. Appl. Physiol. 108, 891-897. doi: 10.1152/japplphysiol.01371.2009

Hatano, N., Suzuki, H., Muraki, Y., and Muraki, K. (2013). Stimulation of human TRPAl channels by clinical concentrations of the antirheumatic drug auranofin. Am. J. Physiol. Cell Physiol. 304, C354-C361. doi: 10.1152/ajpcell.00096.2012

Hinman, A., Chuang, H., Bautista, D. M., and Julius, D. (2006). TRP channel activation by reversible covalent modification. Proc. Natl. Acad. Sci. U.S.A. 103, 19564-19568. doi: 10.1073/pnas.0609598103

Hu, H., Bandell, M., Petrus, M. J., Zhu, M. X., and Patapoutian, A. (2009a). Zinc activates damage-sensing TRPA1 ion channels. Nat. Chem. Biol. 5, 183-190. doi: $10.1038 /$ nchembio. 146

Hu, H., Grandl, J., Bandell, M., Petrus, M., and Patapoutian, A. (2009b). Two amino acid residues determine 2-APB sensitivity of the ion channels TRPV3 and TRPV4. Proc. Natl. Acad. Sci. U.S.A. 106, 1626-1631. doi: $10.1073 /$ pnas.0812209106

Ibarra, Y., and Blair, N. T. (2013). Benzoquinone reveals a cysteine-dependent desensitization mechanism of TRPA1. Mol. Pharmacol. 83, 1120-1132. doi: 10.1124/mol.112.084194

Jordt, S. E., Bautista, D. M., Chuang, H., McKemy, D. D., Zygmunt, P. M., Högestätt, E. D., et al. (2004). Mustard oils and cannabinoids excite sensory nerve fibres through the TRP channel ANKTM1. Nature 427, 260-265. doi: 10.1038 /nature 02282

Joseph, E. K., and Levine, J. D. (2009). Comparison of oxaliplatin- and cisplatininduced painful peripheral neuropathy in the rat. J. Pain 10, 534-541. doi: 10.1016/j.jpain.2008.12.003

Kozai, D., Kabasawa, Y., Ebert, M., Kiyonaka, S., Otani, Y., Numata, T., et al. (2014). Transnitrosylation directs TRPAl selectivity in N-nitrosamine activators. Mol. Pharmacol. 85, 175-185. doi: 10.1124/mol.113.088864

Macpherson, L. J., Dubin, A. E., Evans, M. J., Marr, F., Schultz, P. G., Cravatt, B. F., et al. (2007). Noxious compounds activate TRPA1 ion channels through covalent modification of cysteines. Nature 445, 541-545. doi: $10.1038 /$ nature 05544

Materazzi, S., Fusi, C., Benemei, S., Pedretti, P., Patacchini, R., Nilius, B., et al. (2012). TRPA1 and TRPV4 mediate paclitaxel-induced peripheral neuropathy in mice via a glutathione-sensitive mechanism. Pflugers Arch. 463, 561-569. doi: 10.1007/s00424-011-1071-x

Miller, E. W., Tulyathan, O., Tulyanthan, O., Isacoff, E. Y., and Chang, C. J. (2007). Molecular imaging of hydrogen peroxide produced for cell signaling. Nat. Chem. Biol. 3, 263-267. doi: 10.1038/nchembio871

Miltenburg, N. C., and Boogerd, W. (2014). Chemotherapy-induced neuropathy: a comprehensive survey. Cancer Treat. Rev. 40, 872-882. doi: 10.1016/j.ctrv.2014.04.004

Miura, S., Takahashi, K., Imagawa, T., Uchida, K., Saito, S., Tominaga, M., et al. (2013). Involvement of TRPA1 activation in acute pain induced by cadmium in mice. Mol. Pain 9:7. doi: 10.1186/1744-8069-9-7

Miyake, T., Nakamura, S., Zhao, M., So, K., Inoue, K., Numata, T., et al. (2016). Cold sensitivity of TRPA1 is unveiled by the prolyl hydroxylation blockade-induced sensitization to ROS. Nat. Commun. 7:12840. doi: $10.1038 /$ ncomms12840

Nassini, R., Gees, M., Harrison, S., De Siena, G., Materazzi, S., Moretto, N., et al. (2011). Oxaliplatin elicits mechanical and cold allodynia in rodents via TRPA1 receptor stimulation. Pain 152, 1621-1631. doi: 10.1016/j.pain.2011.02.051
Old, E. A., Nadkarni, S., Grist, J., Gentry, C., Bevan, S., Kim, K. W., et al. (2014) Monocytes expressing CX3CR1 orchestrate the development of vincristineinduced pain. J. Clin. Invest. 124, 2023-2036. doi: 10.1172/JCI71389

Screnci, D., McKeage, M. J., Galettis, P., Hambley, T. W., Palmer, B. D., and Baguley, B. C. (2000). Relationships between hydrophobicity, reactivity, accumulation and peripheral nerve toxicity of a series of platinum drugs. $\mathrm{Br}$. J. Cancer 82, 966-972. doi: 10.1054/bjoc. 1999.1026

Sittl, R., Lampert, A., Huth, T., Schuy, E. T., Link, A. S., Fleckenstein, J., et al. (2012). Anticancer drug oxaliplatin induces acute coolingaggravated neuropathy via sodium channel subtype NaV1.6-resurgent and persistent current. Proc. Natl. Acad. Sci. U.S.A. 109, 6704-6709. doi: 10.1073/pnas.1118058109

So, K., Tei, Y., Zhao, M., Miyake, T., Hiyama, H., Shirakawa, H., et al. (2016). Hypoxia-induced sensitisation of TRPA1 in painful dysesthesia evoked by transient hindlimb ischemia/reperfusion in mice. Sci. Rep. 6:23261. doi: 10.1038/srep23261

Takahashi, N., Kuwaki, T., Kiyonaka, S., Numata, T., Kozai, D., Mizuno, Y., et al. (2011). TRPA1 underlies a sensing mechanism for $\mathrm{O}_{2}$. Nat. Chem. Biol. 7, 701-711. doi: 10.1038/nchembio.640

Takahashi, N., Mizuno, Y., Kozai, D., Yamamoto, S., Kiyonaka, S., Shibata, T., et al. (2008). Molecular characterization of TRPA1 channel activation by cysteine-reactive inflammatory mediators. Channels 2, 287-298. doi: $10.4161 /$ chan.2.4.6745

Toyama, S., Shimoyama, N., Ishida, Y., Koyasu, T., Szeto, H. H., and Shimoyama, M. (2014). Characterization of acute and chronic neuropathies induced by oxaliplatin in mice and differential effects of a novel mitochondriatargeted antioxidant on the neuropathies. Anesthesiology 120, 459-473. doi: 10.1097/01.anes.0000435634.34709.65

Trevisan, G., Materazzi, S., Fusi, C., Altomare, A., Aldini, G., Lodovici, M., et al. (2013). Novel therapeutic strategy to prevent chemotherapy-induced persistent sensory neuropathy by TRPA1 blockade. Cancer Res. 73, 3120-3131. doi: 10.1158/0008-5472.CAN-12-4370

Viana, F. (2016). TRPA1 channels: molecular sentinels of cellular stress and tissue damage. J. Physiol. 594, 4151-4169. doi: 10.1113/JP270935

Wilson, B. R. H., Lehky, T., Thomas, R. R., Quinn, M. G., Floeter, M. K., and Grem, J. L. (2002). Acute oxaliplatin-induced peripheral nerve hyperexcitability. J. Clin. Oncol. 20, 1767-1774. doi: 10.1200/JCO.2002.07.056

Wu, L., Sweet, T., and Clapham, D. (2010). International Union of basic and clinical pharmacology. LXXVI. Current progress in the mammalian TRP ion channel family. Pharmacol. Rev. 62, 381-404. doi: 10.1124/pr.110. 002725

Zhao, M., Isami, K., Nakamura, S., Shirakawa, H., Nakagawa, T., and Kaneko, S. (2012). Acute cold hypersensitivity characteristically induced by oxaliplatin is caused by the enhanced responsiveness of TRPA1 in mice. Mol. Pain 8:55. doi: 10.1186/1744-8069-8-55

Zheng, H., Xiao, W. H., and Bennett, G. J. (2011). Functional deficits in peripheral nerve mitochondria in rats with paclitaxel- and oxaliplatinevoked painful peripheral neuropathy. Exp. Neurol. 232, 154-161. doi: 10.1016/j.expneurol.2011.08.016

Conflict of Interest Statement: The authors declare that the research was conducted in the absence of any commercial or financial relationships that could be construed as a potential conflict of interest.

Copyright (C) 2017 Miyake, Nakamura, Meng, Hamano, Inoue, Numata, Takahashi Nagayasu, Shirakawa, Mori, Nakagawa and Kaneko. This is an open-access article distributed under the terms of the Creative Commons Attribution License (CC $B Y)$. The use, distribution or reproduction in other forums is permitted, provided the original author(s) or licensor are credited and that the original publication in this journal is cited, in accordance with accepted academic practice. No use, distribution or reproduction is permitted which does not comply with these terms. 